\title{
TUMORES DE UROTELIO SUPERIOR
}

\author{
J.A. PORTILLO MARTÍN, M.A. RADO VELÁZQUEZ, J.L. GUTIÉRREZ BAÑOS, \\ M.A. CORREAS GÓMEZ, R. HERNÁNDEZ RODRÍGUEZ, J.I. DEL VALLE SCHAAN, \\ A. ROCA EDREIRA, A. HERNÁNDEZ CASTRILLO, F. RUIZ IZQUIERDO, \\ C. AGUILERA TUBET
}

Servicio de Urología. Hospital Universitario Valdecilla. Facultad de Medicina. Universidad de Cantabria. Santander.

Actas Urol Esp. 28 (1): 7-12, 2004

\section{RESUMEN}

TUMORES DE UROTELIO SUPERIOR

OBJETIVOS: Evaluar los métodos diagnósticos empleados, el tratamiento y el seguimiento de los pacientes afectos de tumor de urotelio superior.

PACIENTES Y MÉTODO: Desde 1978 hasta diciembre de 2002 han sido operados 105 pacientes afectos de tumor de urotelio superior, siendo válidos para el análisis 94 . La edad media ha sido de 65 años, siendo el 85\% varones. La hematuria fue el síntoma más frecuente de presentación.

RESULTADOS: Se realizó UIV en el 93\% de los pacientes, la ecografía en el 77\% y el TAC en el $67 \%$. Predominó el tumor piélico (71\%) y el tratamiento mayoritario fue la nefroureterectomía total con resección del collarete vesical perimeatal $(76,4 \%)$. El tumor vesical previo o simultáneo se constató en 22 casos $(23,3 \%)$ y posterior en 28 casos $(30 \%)$. Con un seguimiento medio de 76 meses, la supervivencia es del $53 \%$.

CONCLUSIONES: El tumor de urotelio superior debe considerarse como una enfermedad panurotelial debido a la alta frecuencia de tumor vesical previo, simultáneo o posterior, lo cual empeora aún más el pronóstico de esta enfermedad.

PALABRAS CLAVES: Tumor urotelio superior. Enfermedad panurotelial. Nefroureterectomía total.

\section{ABSTRACT}

\section{UPPER URINARY TRACT TUMOURS}

OBJECTIVE: To evaluate diagnostic techniques, treatment and follow-up in 94 patients affected of upper urinary tract tumor.

PATIENTS AND METHOD: From 1978 to december 2002 we operated 105 patients due to upper urinary tract tumor, although only 94 are valid for analysis. Mean age was 65 years and $85 \%$ were man. Haematuria was the most frequent symptom.

RESULTS: Urography (93\%), ecography (77\%) and CT (67\%) were the most used diagnostic techniques. Pelvic tumor was the most frequent $(71 \%)$ and total nephroureterectomy including bladder cuff the chosen treatment (76.4\%). Previous or simultaneous bladder tumor was observed in $23 \%$ cases and delayed in $30 \%$. With a mean follow-up of 76 months the patient survival is $53 \%$.

CONCLUSIONS: Due to the high frequence of previous, simultaneous or delayed bladder tumors, the upper urinary tract tumor should be considered as a panurothelial disease, worsening the outcome of this kind of tumors. 
L os tumores de urotelio superior (TUS) son muy poco frecuentes (menos del 5\%) entre todos los tumores uroteliales. Estos tumores se pueden presentar de forma aislada, o bien relacionados con un tumor vesical, siendo su diagnóstico previo, simultáneo o posterior a dicho tumor vesical.

Debido a la multifocalidad de los tumores uroteliales en general, una vez diagnosticado no debe olvidarse en el seguimiento el estudio de todo el urotelio para descartar otros implantes diferentes al original.

El tratamiento clásico ha sido la nefroureterectomía total con extirpación del collarete vesical perimeático. No obstante, el tratamiento endourológico conservador también tiene su papel y no sólo cuando hablamos de riñones únicos.

A continuación referimos nuestra experiencia con este tipo de tumores en nuestro hospital.

\section{PACIENTES Y MÉTODO}

Desde 1978 hasta diciembre de 2002 han sido intervenidos en nuestro Servicio de Urología del Hospital Universitario Valdecilla de Santander 105 pacientes afectos de TUS, siendo válidos para el estudio 94, en edades comprendidas entre los 29 y los 86 años (edad media de 65), de ellos 80 varones y 14 mujeres.

De los 94 pacientes válidos, se han recogido datos referidos a la sintomatología, antecedentes tabáquicos, métodos diagnósticos, tratamiento efectuado, relación con tumores uroteliales de otra localización y seguimiento.

El método estadístico empleado ha sido con el programa SPSS 8.0 versión para Windows

\section{RESULTADOS}

De los 94 pacientes, en 79 se pudo recoger el antecedente tabáquico. De ellos 22 (28\%) no habían fumado nunca, 18 (23\%) fumaban menos de 20 cigarrillos y 39 (49\%) más de 20 al día.

Mayoritariamente (el 87\%) se había presentado con hematuria sobre todo macroscópica, acompañada con cólico renal o no. En otros casos con infección urinaria (2\%), cólico aislado (6\%) o hallazgo casual (5\%) en el curso de otra patología.

Los métodos diagnósticos empleados han sido la ecografía (77\%), la UIV (93\%), el TAC (67\%) y en un caso anecdótico reciente la RNM.
La pielografía ascendente se consideró necesaria en el $25 \%$ de los casos. En 8 casos portadores de nefrostomía debido a obstrucción urinaria, se practicó asimismo pielografía descendente.

La citología urinaria se recogió en el 53\% de los pacientes, en la mayoría por vaciado espontáneo vesical, siendo el $40,7 \%$ positiva y el $12,3 \%$ negativa. Sobre todo fue positiva en los de mayor grado histológico.

En 17 pacientes (18\%) se había diagnosticado un tumor vesical previo y en otros 5 (5,3\%) el tumor vesical fue simultáneo. De los 22 casos, en 20 se practicó RTU, en 1 cistectomía parcial y en el otro cistectomía radical. El diagnóstico anatomo-patológico de los 22 casos fue en 17 pT1G2, en 3 pT1G3 y en los otros 2 pT2G3.

Tras los métodos diagnósticos empleados, se llegó al diagnóstico preoperatorio de 67 tumores piélicos, 23 ureterales y 4 caliciales, lo cual tuvo una correlación casi exacta con el análisis de la pieza (Tabla I).

En 51 casos fueron derechos (54,3\%) y en 43 izquierdos.

La técnica quirúrgica ha sido predominantemente la nefroureterectomía total con resección del collarete vesical perimeatal (72 casos, 76,4\%). De ellos en 57 casos se utilizó la doble incisión extraperitoneal y en otros 2 se desinsertó el uréter endoscópicamente. En los otros 22 se practicó nefrectomía simple, sin resección de todo el uréter.

$\mathrm{El}$ tamaño de la neo ha variado entre los $9 \mathrm{~mm}$ y los $11 \mathrm{~cm}$, siendo el tamaño medio de $3 \mathrm{~cm}$.

El estudio histológico reveló que en 49 casos $(52,1 \%)$ el tumor estaba limitado a la mucosa o submucosa (Ta-T1), en 10 casos $(10,7 \%)$ infiltraba la muscular (T2), en $18(19,1 \%)$ infiltraba la grasa perirrenal, y en $17(18,1 \%)$ infiltraba el parénquima renal (T3) (Tabla II).

En cuanto al grado histológico, 3 fueron Grado I (3,2\%), 49 Grado II $(52,1 \%)$ y 42 Grado III $(44,7 \%)$ (Tabla III).

\section{TABLA I}

LOCALIZACIÓN DEL TUMOR

\begin{tabular}{|l|c|c|c|}
\hline & Calicial & Piélica & Ureteral \\
\hline Pre I.Q. & 4 & 67 & 23 \\
\hline A-P & 3 & 68 & 23 \\
\hline
\end{tabular}


TABLA II

INFILTRACIÓN DEL TUMOR

\begin{tabular}{|l|c|}
\hline & № de casos \\
\hline Mucosa-Submucosa & $49-52,1 \%$ \\
\hline Muscular & $10-10,7 \%$ \\
\hline Grasa perirrenal & $18-19,1 \%$ \\
\hline Parénquima renal & $17-18,1 \%$ \\
\hline
\end{tabular}

TABLA III

GRADO DEL TUMOR

\begin{tabular}{|c|c|}
\hline Grado & № de casos \\
\hline I & $3-3,2 \%$ \\
\hline II & $49-52,1 \%$ \\
\hline III & $42-44,7 \%$ \\
\hline
\end{tabular}

En la mayoría de los pacientes no se aplicó otro tratamiento excepto en 4 casos a los que por diversas circunstancias se les aplicó quimioterapia post-cirugía.

En 28 pacientes (30\%) se desarrolló tumor vesical en el seguimiento, variando desde una única recidiva (15 casos), dos recidivas (8 casos), a incluso 11 recidivas en un caso. La aparición del tumor vesical varió desde los 2 meses incluso hasta los 88 meses tras la nefrectomía. En la mayoría se practicó RTU unido a lavados vesicales con quimioterápicos locales. En tres casos se realizó finalmente cistectomía por mal control local de la enfermedad o por hacerse el tumor infiltrante de la muscular.

En 8 casos tenemos constancia de recidiva local, bien como tal o por ser tumor residual por invasión local, realizando solamente cirugía de rescate en uno de ellos.

En 20 casos $(21,5 \%)$ se presentaron metástasis en el seguimiento, en 9 casos múltiples, en 4 pulmonares, en 5 óseas y en 2 hepáticas.

Con un seguimiento medio de 76 meses (7237), 50 pacientes (53\%) se encuentran vivos, de ellos 7 con algún tipo de enfermedad urotelial. Otros 42 han fallecido, la mayoría debido a la enfermedad en estudio y dos están perdidos para el seguimiento. Datos parecidos a otras series publicadas (Tablas IV y V), aunque son dificiles de comparar ya que se mezclan diversos grados, estadios y tratamientos aplicados.

\section{TABLA IV}

\begin{tabular}{||l|c|c|c|c||}
\hline Autor & № casos & Seguimiento & Supervivencia & Tratamiento \\
\hline $\begin{array}{l}\text { Charbit } \\
\text { (1991) }\end{array}$ & 108 & 10 A. & $65 \%$ & Radical \\
\hline $\begin{array}{l}\text { Ruiz-Cerdá } \\
\text { (1993) }\end{array}$ & 92 & 10 A. & $45 \%$ & $64 \%$ radical \\
\hline $\begin{array}{l}\text { Salinas } \\
\text { (1995) }\end{array}$ & 35 & 5 A. & $60 \%$ & $57 \%$ radical \\
\hline $\begin{array}{l}\text { Shie-Herng } \\
\text { (1996) }\end{array}$ & 68 & 5 A. & $\begin{array}{l}\text { Bajo gr.: 93,6\% } \\
\text { Alto gr.: 28,3\% }\end{array}$ & Radical \\
\hline $\begin{array}{l}\text { Maulard } \\
\text { (1996) }\end{array}$ & 26 & 45 M. & $49 \%$ & Radical + RT \\
\hline \hline
\end{tabular}

\section{TABLA V}

COMPARACIÓN DE SERIES (2)

\begin{tabular}{|l|c|c|c|c|}
\hline Autor & № casos & Seguimiento & Supervivencia & Tratamiento \\
\hline $\begin{array}{l}\text { Racioppi } \\
\text { (1997) }\end{array}$ & 100 & $15 \mathrm{~A}$. & $\begin{array}{l}69 \% \\
25 \%\end{array}$ & $\begin{array}{c}\text { Radical } \\
\text { Conserv. }\end{array}$ \\
\hline $\begin{array}{l}\text { Ferrero } \\
(1998)\end{array}$ & 60 & $3,5 \mathrm{~A}$. & $\begin{array}{c}\text { Est. O-A: 96\% } \\
\text { Est. B-D: } 44 \%\end{array}$ & $\begin{array}{c}34 \% \text { Conserv. } \\
64 \% \text { Radical }\end{array}$ \\
\hline $\begin{array}{l}\text { Arrizabalaga } \\
(1998)\end{array}$ & 50 & $5 \mathrm{~A}$. & $61 \%$ & $\begin{array}{c}40 \% \text { Conserv. } \\
60 \% \text { Radical }\end{array}$ \\
\hline $\begin{array}{l}\text { Deligne } \\
(2002)\end{array}$ & 61 & $40 \mathrm{M}$. & $84 \%$ & Conserv. \\
\hline $\begin{array}{l}\text { Valdecilla } \\
(2003)\end{array}$ & 94 & $76 \mathrm{M}$. & $53 \%$ & Radical \\
\hline \hline
\end{tabular}

\section{DISCUSION}

Llamamos urotelio superior al epitelio transicional que recubre las superficies de cálices, pelvis y uréter hasta su entrada en vejiga. Debido a que el mismo epitelio recubre la vejiga y parte de uretra, los tumores que se desarrollan en todas estas estructuras tienen características muy similares.

Es de sobra conocida la multifocalidad del tumor vesical. De la misma forma a los TUS los debemos considerar como tumores posiblemente multifocales, y por tanto vigilar todo el epitelio transicional para descartar otras localizaciones tumorales.

Se han estudiado los factores pronósticos que pudieran influir en el desarrollo de TUS tras padecer un tumor vesical. Para Millán lo más influyente parece ser la multifocalidad del tumor primario ${ }^{1}$, incluso por encima del grado, estadio, tamaño tumoral, cis asociado o las instilaciones intravesicales. Sin embargo, para Hurle ${ }^{2}$ el cis y 
los de Grado III tienen más posibilidades de desarrollar TUS. También Solsona ${ }^{3}$ y Schwartz S $^{4}$ observan que el cis vesical es un factor pronóstico influyente.

Aunque el mayor riesgo de TUS post-ca. vesical es en los 2-3 primeros años ${ }^{5}$, parece lógico que a mayor seguimiento, mayor riesgo de desarrollo de TUS. Obdring observa esta circunstancia incluso a los 13 años de seguimiento ${ }^{6}$, Herr a los $15^{7}$ y Schwartz a los 20 años $^{4}$. Por tanto el seguimiento a largo plazo de estos pacientes es obligado, vigilando el urotelio superior sobre todo con UIV ${ }^{8}$.

En un meta-análisis de 5.461 pacientes con ca. vesical, el 1,96\% desarrolló TUS metacrónico con un seguimiento medio de 58 meses. Por contra de 742 pacientes con TUS, el 24\% desarrolló ca. vesical metacrónico con un seguimiento medio de 18,9 meses $^{5}$. El grado de ambos tumores suele ser el mismo. Sekine ${ }^{9}$ y Charbit ${ }^{10}$ observan una parecida incidencia. Angulo ${ }^{11}$ refiere que de 338 tumores vesicales, el $4 \%$ desarrolla TUS, señalando una menor supervivencia cuando los tumores son sincrónicos.

Los factores de riesgo que más parecen influir en el desarrollo de ca. vesical post-TUS son la multifocalidad, el grado y el estadio patológico ${ }^{12,15}$.

La anulación funcional también se ha postulado como factor influyente, al menos en la supervivencia ${ }^{16}$.

Otro factor estudiado ha sido la ploidia, observando Baladament en el análisis multivariante que los tumores aneuploides tenían una menor supervivencia ${ }^{17}$, aunque en la supervivencia global el único factor influyente fue el estadio. Lee también determinó la ploidia de los TUS, no encontrando diferencias entre los diferentes estadios y $\operatorname{grados}^{18}$.

La forma de presentación más frecuente es la hematuria, aunque debido a su localización también puede causar cólico renal, dolor sordo lumbar e incluso fiebre asociada a infección urinaria.

$\mathrm{El}$ diagnóstico por imágenes no es difícil con la ecografía, urografía, TAC o incluso recurriendo a la pielografía ascendente o a la descendente ante riñones obstruidos. La citología es de menor ayuda, siendo sobre todo útil si es persistentemente positiva, sobre todo en tumores de alto grado.
La presencia de un tumor del urotelio superior plantea no sólo el problema de su tratamiento en función de si se debe ser conservador o no, sino que además debemos ser conscientes de que nos enfrentamos ante una enfermedad multifocal o panurotelial.

La tendencia clásica ha sido el tratar a estos tumores de forma radical, con la nefroureterectomía total. Se extirpa asimismo la zona vesical perimeatal, (habitualmente por medio de la doble incisión extraperitoneal), debido al alto índice de recidivas a ese nivel, aunque algunos autores no comparten este criterio $^{19}$. También se puede desinsertar endoscópicamente el uréter y por lumbotomía extirpar toda la pieza, evitando la doble incisión ${ }^{20}$.

El reto se plantea en pacientes monorrenos en los que se debe ser razonablemente conservador para no conducir al paciente a situación definitiva de diálisis.

Con el desarrollo de la endourología, tanto con la ureteroscopia como con la nefroscopia, se ha conseguido abordar estos tumores de forma conservadora, asumiendo el riesgo de la recidiva posterior. En los últimos años no sólo se han aplicado tratamientos conservadores a riñones únicos sino que también a pacientes con dos riñones, siempre que el tamaño, la localización y el grado del tumor así lo permitieran. Otros autores no son partidarios de este tipo de abordaje terapéutico en ningún caso debido precisamente a la multifocalidad $^{10}$.

Deligne $^{21}$ resuelve endoscópicamente 61 casos de TUS, realizando posteriormente en 6 casos nefrectomía ( $81 \%$ de conservación renal), con una supervivencia del $84 \%$ a los 40 meses de seguimiento. También Elliot ${ }^{22}$ obtiene un $81 \%$ de conservación renal en 21 casos, aunque con un $46 \%$ de recidivas de tumores ureterales y un $12 \%$ de los tumores piélicos.

Chen ${ }^{23}$ resuelve 23 tumores ureterales de bajo grado por medio de la ureteroscopia, pero con el $64 \%$ de recidivas. Martínez-Piñeiro ${ }^{24}$ en 59 casos de tratamiento endoscópico de TUS obtiene a los 30 meses de seguimiento un $23,8 \%$ de recurrencias.

Racioppi ${ }^{25}$ compara dos grupos de pacientes (en total 100), en los que aplica en el 53\% nefroureterectomía clásica y en el $47 \%$ tratamiento con- 
servador (nefrectomía parcial, tumorectomías o ureterectomías). El indice de recidivas en el primer grupo es de $40 \%$ y en el otro del $70 \%$. Asimismo con el tratamiento clásico la supervivencia a los 15 años es del 69\% y en el otro grupo es del $25 \%$. Otros autores también observan mayor índice de recidivas con el tratamiento conservador por lo que recomiendan aplicarlo sólo en los tumores de bajo grado ${ }^{11,17,26-28}$.

Se ha utilizado la radioterapia (RT) como complemento de la cirugía, observando una supervivencia similar a la de otras series sin RT, por tanto no se recomienda como tratamiento adicional a la cirugía ${ }^{29}$. Otros autores creen que la RT tiene un papel en los TUS de grado III ya que parece que disminuye algo el índice de recidivas, pero sin prolongar la supervivencia ${ }^{30}$.

La conclusión que extraemos de los casos publicados es que el tratamiento clásico sigue teniendo vigencia, y que el tratamiento endourológico conservador en principio tiene su máxima aplicación en los tumores de bajo grado y en los monorrenos.

\section{REFERENCIAS}

1. MILLÁN F, CHECHILE G, SALVADOR-BAYARRI J, HUGUET J, VICENTE J.: Upper urinary tract tumors after primary superficial bladder tumors: prognostic factors and risk groups. J Urol 2000; 164: 11831187.

2. HURLE R, LOSA A, MANZETTI A, LEMBO A.: Upper urinary tract tumors developing after treatment of superficial bladder cancer: 7-year follow-up of 591 consecutive patients. Urology 1999; 53: 1144-1148.

3. SOLSONA E, IBORRA I, RICOS JV, DUMONT R, CASANOVA JL, CALABUIG C.: Upper urinary tract involvement in patients with bladder carcinoma in situ (Tis): its impact on management. Urology 1997; 49: 347-352.

4. SCHWARTZ CB, BEKIROV $\mathrm{H}$, MELMAN A.: Urothelial tumors of upper tract following treatment of primary bladder transitional cell carcinoma. Urology 1992; 40 (6): 509-511.

5. ARRIZABALAGA M, NAVARRO J, MORA M y cols. Carcinomas transicionales del tracto urinario: lesiones sincrónicas y metacrónicas. Act Urol Esp 1994; 18 (8): 782-796.

6. OLDBRING J, GLIFBERG I, MIKULOWSKI P, HELLSTEN S.: Carcinoma of the renal pelvis and ureter following bladder carcinoma: frequency, risk factors and clinicopathological findings. $J$ Urol 1989; 141: 1311-1313.

7. HERR HW, COOKSON MS, SOLOWAY SM.: Upper tract tumors in patients with primary bladder cancer followed for 15 years. J Urol 1996; 156: 1286-1287.
8. HUGUET J, PALOU J, MILLÁN F, SALVADORBAYARRI J, VILLAVICENCIO H, VICENTE J.: Upper tract transitional cell carcinoma following cystectomy for bladder cancer. Eur Urol 2001; 40: 318 323.

9. SEKINE H, FUKUI I, YAMADA T, KIHARA K, ISHIZAKA K, OHSHIMA H.: Clinicopathological study of upper urinary tract tumors associated with bladder tumors. Eur Urol 1991; 19: 19-23.

10. CHARBIT L, GENDREAU MC, MEE S, CUKIER J.: Tumors of the upper urinary tract: 10 years of experience. J Urol 1991; 146: 1243-1246.

11. ANGULO J, ESCRIBANO J, TAMAYO JC, DEHAINI A, GUILY M, SÁNCHEZ-CHAPADO M.: Tumores de tracto urinario superior y uretra en pacientes con carcinoma vesical. Arch Esp Urol 1997; 50 (2): 115120.

12. HISATAKI T, MIYAO N, MASUMORI N et al.: Risk factors for the development of bladder cancer after upper tract urothelial cancer. Urology 2000; 55: 663-667.

13. HALL MG, WOMACK S, SAGALOWSKY AI, CARMODY T, ERICKSTAD MD, ROEHRBORN CG.: Prognostic factors, recurrence and survival in transitional cell carcinoma of the upper urinary tract: a 30-year experience in 252 patients. Urology 1998; 52: $594-601$.

14. FERRERO R, GUZMÁN PL, MORGA JP y cols.: Tumores de tracto urinario superior. Act Urol Esp 1998; 22 (5): 410-416.

15. SALINAS AS, HERNÁNDEZ IR, SEGURA M y cols.: Estudio de supervivencia en pacientes con tumor de urotelio superior. Arch Esp Urol 1995; 48 (7): 688-700.

16. RUIZ-CERDÁ JL, MORERA J, PONTONES JL y cols.: Factores pronósticos de los tumores del tracto urinario superior. Act Urol Esp 1993; 17 (9): 547-554.

17. BADALAMENT RA, O`TOOLE RV, KENWORTHY P et al.: Prognostic factors in patients with primary transitional cell carcinoma of the upper urinary tract. J Urol 1990; 144: 859-863.

18. SHIE-HERNG L, SHINN-NAN J, TZONG-SHIN T et al.: Prognostic factors of primary transitional cell carcinoma of the upper urinary tract. Eur Urol 1996; 29: 266-271.

19. BEGARA F, RAMÍREZ JC, HERMIDA J y cols.: Tumores uroteliales del tracto urinario superior: estudio epidemiológico, evaluación diagnóstica y terapéutica. Arch Esp Urol 1996; 49 (1): 27-33.

20. SALVADOR-BAYARRI J, RODRÍGUEZ-VILLAMIL L, IMPERATORE V, PALOU J, VILLAVICENCIO $\mathrm{H}$, VICENTE J.: Bladder neoplasms after nephroureterectomy: does the surgery of the lower ureter, transurethral resection or open surgery, influence the evolution?. Eur Urol 2002; 41: 30-33.

21. DELIGNe E, COLOMBEL M, BADET L et al.: Conservative management of upper urinary tract tumors. Eur Urol 2002; 42: 43-48.

22. ELLIOT DS, SEGURA JW, LIGHTNER D, PATTERSON DE, BLUTTE ML.: Is nephroureterectomy necessary in all cases of upper tract transitional cell carcinoma? Long-term results of conservative 
endourologic management of upper tract transitional cell carcinoma in individuals with a normal contralateral kidney. Urology 2001; 58: 174-178.

23. CHEN GL, EL-GABRY A, BAGLEY DH.: Surveillance of upper urinary tract transitional cell carcinoma: the role of ureteroscopy, retrograde pyelography, cytology and urinalysis. J Urol 2000; 164: 1901-1904.

24. MARTÍNEZ-PIÑEIRO JA, GARCÍA MJ, MARTÍNEZPIÑEIRO L.: Endourological treatment of upper tract urothelial carcinomas: analysis of a series of 59 tumors. J Urol 1996; 156: 377-385.

25. RACIOPPI M, D ADDESSI A, ALCINI A, DESTITO A, ALCINI E.: Clinical review of 100 consecutive surgically treated patients with upper urinary tract transitional tumors. Br J Urol 1997; 80: 707-711.

26. MUÑOZ D, REBASSA M, HIDALGO F y cols.: Tumores del tracto urinario superior: resultados del tratamiento y seguimiento. Arch Esp Urol 1999; 52 (4): 333-341.

27. ARRIZABALAGA M, DIEZ JM, GARCÍA LI y cols.: Carcinomas uroteliales del tracto urinario superior. Supervivencia y factores pronósticos. Arch Esp Urol 1998; 51 (3): 243-251.
28. KROGH J, KVIST E, RYE B.: Transitional cell carcinoma of the upper urinary tract: prognostic variables and post-operative recurrences. $\mathrm{Br} \mathrm{J} \mathrm{Urol}$ 1991; 67: 32-36.

29. MAULARD-DURDUX C, DUFOUR B, HENNEGUIN $\mathrm{C}$ et al.: Postoperative radiation therapyin 26 patients with invasive transitional cell carcinoma of the upper urinary tract: no impact on survival?. $J$ Urol 1996; 155: 115-117.

30. FERNÁNDEZ A, MARTÍNEZ JL, DOMÍNGUEZ JF y cols.: Eficacia de la radioterapia en los tumores del tracto urinario superior tras cirugía radical. Arch Esp Urol 1998; 51 (7): 695-700.

Dr. J.A. Portillo Martín

Avda. del Faro, 1 - Bl. 2, 3ํㅡ

39012 Santander (Cantabria)

(Trabajo recibido el 2 septiembre de 2003) 